\title{
Reconocimiento y espacialización de Unidades Territoriales Residenciales (UTR) en la circunscripción IV - Sección B. Río Gallegos
}

\section{Land occupation process with residential use in Circumscription IV - Section B, Río Gallegos, Southern Patagonia Argentina}

\author{
Merlina L. Saldivia, Alicia P. Cáceres, Cristian A. Ampuero \\ merlinasaldivia.22@gmail.com,acaceres@uarg.unpa.edu.ar,cristianampuero@gmail.com \\ Universidad Nacional de la Patagonia Austral (UNPA), Unidad Académica Río Gallegos \\ (UARG), Instituto de Ciencias del Ambiente, Sustentabilidad y Recursos Naturales \\ (ICASUR), Grupo Z Terra Cognita.
}

Recibido: 01/07/2019. Aceptado: 20/11/2019

\section{RESUMEN}

Este trabajo es producto de una beca de investigación CIN, la cual tuvo como finalidad reconocer y espacializar las Unidades Territoriales Residenciales (UTR) de la Circunscripción IV - Sección B de la Tercera Franja Residencial (TRF) de la ciudad de Río Gallegos, provincia de Santa Cruz, Argentina. Para llevar a cabo esta investigación se recurrió a la observación directa a través de salidas de campo, lectura de bibliografía científica, relevamiento de ordenanzas, cartografía, fotografías aéreas e imágenes satelitales, para luego procesar la información recolectada a través de un Sistema de Información Geográfica (SIG). Se han identificado nueve UTR presentes en el área de estudio, y se dio cuenta de los elementos que constituyen el Paisaje Urbano del área de estudio, a saber, los usos del suelo, proceso de ocupación, entramado y tipo de vivienda/tipología arquitectónica. Este trabajo aporta a la zonificación del uso del suelo que debe realizar en el área de estudio.

Palabras Clave: Geografía Urbana; Paisaje Urbano; RururbanoIntraejidal; Tercera Franja Residencial; Uso del suelo residencial.

\begin{abstract}
This work is the result of a CIN research grant, which aimed to recognize and spatialize the Residential Territorial Units (UTR) of Circumscription IV - Section B of the Third Residential Strip (TRF) of the city of Río Gallegos, province of Santa Cruz, Argentina. In order to carry out this research, direct observation was made through field trips, reading of scientific bibliography, ordinance surveys, cartography, aerial photographs and satellite images, to then process the information collected through a Geographic Information System. (S.I.G). The UTR present in the study area have been identified nine, and the elements that make up the Urban Landscape of the study area have been identified, namely, land uses, occupation process, framework and type of dwelling / architectural typology. This work contributes to the zoning of the land use that must be done in the study area.
\end{abstract}

Keywords: Urban Geography; Urban Landscape; IntraejidalRururbano; Third Residential Strip; Residential land use. 


\section{INTRODUCCIÓN}

El presente trabajo es producto de la actividad realizada por Merlina Saldivia, alumna de la carrera Licenciatura en Trabajo Social, correspondiente a la Escuela de Trabajo Social de la Universidad Nacional de la Patagonia Austral, Unidad Académica Río Gallegos (UNPA UARG), en el marco de una beca de investigación Estímulo a las Vocaciones Científicas del Consejo Interuniversitario Nacional (CIN) - UNPA, dirigida por Alicia P. Cáceres y Codirigida por Cristian Ampuero. El plan de la beca denominada "Reconocimiento y espacialización de Unidades Territoriales Residenciales (UTR) en la Circunscripción IV Sección B. Tercera Franja Residencial de Río Gallegos", se enmarca en el Proyecto de Investigación 29A/PDTS380 “Tercera Franja Residencial de Río Gallegos: Diagnóstico territorial de áreas con ausencia y presencia de cobertura de servicios. Propuesta de localización de Nodos de Servicios"

Según Cáceres y García (2004) la Estructura Urbana Interna ${ }^{1}$ de la ciudad comprende dos grandes áreas con divisiones propias: Urbana y Rururbana. En cuanto a la primera, se ubica dentro del ejido municipal y comprende al Área Central, Segunda Franja Residencial y Tercera Franja Residencial; mientras que la segunda, comprende parte dentro y fuera del ejido, denominándose Intraejidal y Extraejidal respectivamente. Cabe aclarar que el límite occidental ha variado debido a ampliaciones del ejido municipal entre 2013 y 2018 realizado por la Honorable Cámara de Diputados.

La consolidaciónde la Tercera Franja Residencial (TFR)comienza en el Siglo XX en virtud del proceso de crecimiento demográfico que tuvo como efecto la demanda de tierras para su uso habitacional. Siguiendo a Cáceres (2013)citada en Ampuero, Cáceres, Norambuena, Frias y Melgarejo (2018) son dos hechos los que permitieron la superación de las barreras antrópicas del Borde Urbano y el crecimiento urbano de la ciudad: "En primer lugar, se destaca la recuperación de terrenos de las Fuerzas Armadas hacia el sur y este de la ciudad; y, en segundo lugar, la sustitución del Ramal Ferroindustrial Río Turbio por la Av. Balbín y el cierre del muelle Río Turbio del puerto de Río Gallegos, al oeste de la ciudad." (Ampuero, Cáceres, Norambuena, Frias y Melgarejo, 2018, p.2)

El avance, ocupación y uso del suelo en la TFRen tanto emergente de su contexto, generó contradicciones en las políticas de planificación urbana al exceder los límites impuestos desde la normativa del Estado Municipal para uso residencial del suelo. En este sentido, sostienen Cáceres, Norambuena, Ampuero y Triviño(2013) que "el avance urbano tiene como consecuencia la consolidación del área en la TFR, de modo espontáneo, sin planificación [...], el Estado municipal no reconoce a la franja en su conjunto, hay ausencia de políticas de planificación urbana de modo integrado entre el Estado nacional, provincial y municipal. De este modo, se profundiza el carácter monocéntrico de Río Gallegos, la periferia no ofrece

\footnotetext{
${ }^{1}$ En un estudio sobre la denominada Tercera Franja Residencial, Cáceres et al (2013) sostienen que desde el punto de vista geográfico se considera la definición de Estructura Interna "a partir de análisis de los componentes de la morfología urbana - usos del suelo, tipo de plano, edificación- en relación a los factores de tipo económico, demográfico, político e histórico. ”(Cáceres et al; 2013: 48)

Es decir, que, como concepto, la Estructura Interna permite la identificación de las etapas de crecimiento desde el punto de vista natural y antrópico en cuanto al modo de apropiación y uso del suelo, como así también, los modelos políticos que intervienen en los procesos de urbanización. Esto permite la diferenciación áreas en las ciudades.
} 
equipamiento, infraestructura y servicios". (Cáceres, Norambuena, Ampuero y Triviño, 2013, p. 58).

En cuanto al Área RururbanaIntraejidal ${ }^{2}(\mathrm{ARI})$ está caracterizada por la presencia de chacras subdivididas en lotes de dimensiones urbanas. Acerca de su conformación histórica, Cáceres, Sáenz, Melgarejo y Ampuero (2011)mencionan que:

"En los ochenta, ante la falta de tierras fiscales dentro del área urbana y el alto porcentaje de chacras improductivas, una fuerte especulación inmobiliaria favoreció loteos de dimensiones urbanas vendidos a particulares, cuya ocupación se lleva a cabo con viviendas de uso permanente principalmente y vivienda de carácter secundario. De este modo, la expansión de estos usos fuera del área urbana no le dio al municipio tiempo de ordenar este espacio en forma eficiente por lo tanto genera un crecimiento urbano descontrolado." (Cáceres, Sáenz, Melgarejo y Ampuero, 2011, p. 7)

Siguiendo la misma línea, debe comprenderse que las necesidades de los sujetos son multidimensionales y no se agotan al satisfacer únicamente la demanda habitacional, puesto que el proceso de ocupación del suelo para uso residencial también supone la garantía de otros derechos como parte de las competencias del Estado para la reproducción cotidiana de los ciudadanos: el acceso a una vivienda digna, como así también, el acceso a la salud, a la educación, a la recreación, etc., derechos inalienables y transversales a todos los ciudadanos en pos de generar un ambiente que permita desarrollar las potencialidades desde un enfoque comunitario que constituya la identidad colectiva.

Refiriendo a características que hacen a la vida cotidiana de los ciudadanos que habitan la Tercera Franja Residencial (TFR)y a los Nodos de Servicios ${ }^{3}$, González y Cáceres (2018) citando a Cáceres y Ampuero (2008) sostienen que:

"El crecimiento y ensanchamiento urbano de la ciudad en la TFR generó distancias importantes hacia el centro, situación que se ve agravada por las inclemencias climáticas (bajas temperaturas en invierno y vientos fuertes en verano) y por un servicio de transportes de colectivos deficiente y costoso. El abastecimiento de servicios y equipamientos dependen algunos de la administración provincial, otros de la municipal y se localizan en parcelas cercanas que comprenden unas pocas manzanas, cuya concentración constituyen "Nodos de Servicios" (Cáceres y Ampuero, 2008) que favorecen la descentralización y que atraen otros usos como el comercial.”(González y Cáceres 2018, p. 6)

El Estado municipal tiene dos formas de dividir y denominar los sectores del ejido que no son coincidentes y no se encuentran articulados entre sí. Por un lado, "la designación catastral" que abarca todo el ejido municipal y comprende los aspectos fiscales y de registro dominial de

\footnotetext{
${ }^{2}$ Cáceresy García (2004) sostienen que se caracteriza por "la invasión desorganizada de diferentes usos del suelo" y "por el abandono y subaprovechamiento que predominan en sus parcelas".

${ }^{3}$ Los Nodos de Servicios son definidos por Cáceres y Ampuero (2008) como un conjunto de unas pocas manzanas contiguas localizadas en puntos estratégicos y accesibles en el área al que pertenecen, y que “...comprenden equipamiento en servicios educativos, de salud, recreativos, comerciales, financieros, seguridad, asistencia social y de sanidad, entre otros que mejoren la calidad de vida de los vecinos”. (Cáceres y Ampuero, 2008: 185)
} 
la parcela, estableciendo unidades que, de mayor a menor, son: Circunscripción (7 denominadas en números romanos) y las Secciones (denominadas en letras mayúsculas variando la cantidad según la Circunscripción, las manzanas y las parcelas).

Por este motivo, este trabajo tiene como objetivo reconocer y espacializar las UTR de la Circunscripción IV - Sección B, como aporte al diagnóstico territorial, principalmente relacionado a la zonificación de usos del suelo que debe realizar la Municipalidad.

Los resultados aportan parcialmente al PI PDTS, en el diagnóstico territorial para identificar, entre otros, las áreas ocupadas con usos residenciales, para la posterior zonificación respecto a los terrenos disponibles para la proyección urbanística del municipio.

\section{DESCRIPCIÓN DEL ÁREA DE ESTUDIO}

Río Gallegos es la capital de Santa Cruz y es la ciudad más austral de la Patagonia Continental Argentina. Es una ciudad intermedia que presenta las características de las ciudades de la Patagonia Austral: ocupación reciente y crecimiento demográfico con un importante componente migratorio.

Pertenece al departamento GüerAike que el Censo 2010 del INDEC contabilizó 113.267 habitantes y según el mismo Censo, Río Gallegos contó con 95.796 habitantes.

Para profundizar sobre la caracterización de la ciudad y el área de estudio, es pertinente acudir a los aportes de Cáceres et al. (2016) sobre posición geográfica, al sostener que “...como concepto se refiere a factores geográficos de un espacio concreto, geométrico, físico, natural, social, económico, vivencial de la superficie terrestre. Es decir, como concepto abarcativo que incluye a Sitio, Emplazamiento y Situación a escala local.” (Cáceres et al., 2016, p. 50)

De esta manera, al caracterizar la posición geográfica de la ciudad y el área de estudio pueden conocerse su ubicación según sus coordenadas geográficas - latitud y longitud - el marco natural en el cual está emplazado y los procesos políticos que atraviesan el territorio en cuanto a los usos y apropiación del suelo en un momento histórico dado.

El sitio de Río Gallegos es $51^{\circ} 38^{\prime}$ ' Latitud Sur, y $69^{\circ} 17^{\prime}$ Longitud Oeste, siendo la ciudad más austral de la Patagonia Continental Argentina. (Fig. 1) 


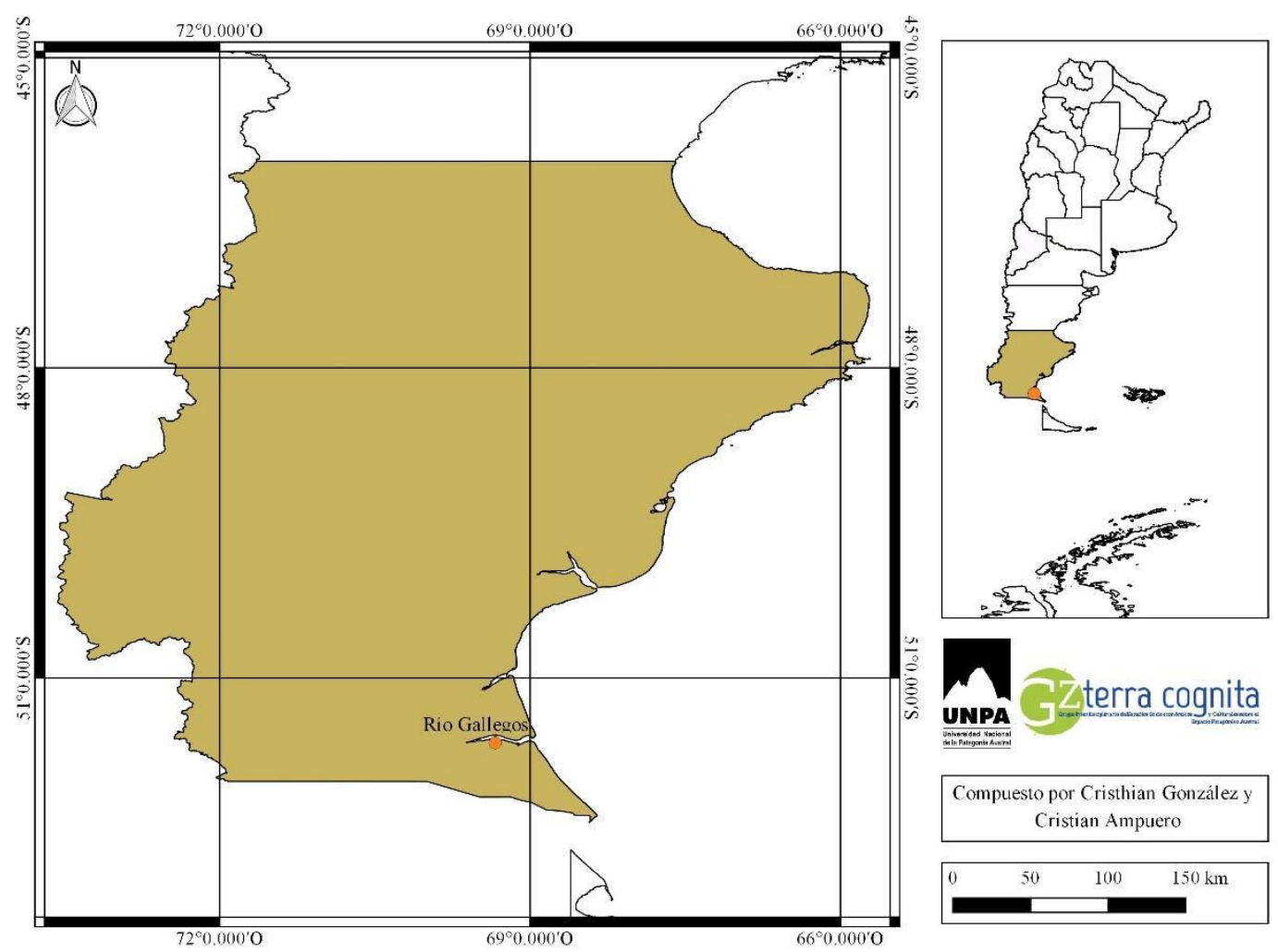

Fig. 1. Posición Geográfica de la ciudad de Río Gallegos. Elaboración propia

Respecto al emplazamiento de Río Gallegos, Cáceres et al. (2013) sostienen que:

“... está emplazada sobre la margen sur del estuario del río Gallegos, sobre terrazas fluviales y algunos paleocauces, a una altura media por debajo de los 20 m.s.n.m. Se encuentra ubicada desde el punto de vista fisiográfico en el denominado Patagonia Austral Extraandina (dentro del ecosistema Estepa Magallánica), con ausencia absoluta de árboles, propio del tipo de clima de esta zona, frío semiárido de meseta con precipitaciones que no superan los $300 \mathrm{~mm}$ anuales. La latitud es un condicionante de la vida urbana puesto que corresponde a la zona de libre circulación atmosférica y oceánica; que explica la presencia de vientos fuertes del Oeste, la falta de verano térmico y los días largos del verano y las noches largas del invierno. Por lo tanto, el emplazamiento se caracteriza por la aridez, vientos fuertes y mesetas de suave relieve que generan un paisaje natural, monótono y hostil." (Cáceres et al., 2013, p. 2)

La Situación de Río Gallegos, según Cáceres et al.(2016) puede ser estudiada en la historia a partir de tres cortes temporales que dan cuenta del contexto particular de la ciudad:

- 1920 a 1960: situación de encrucijada de ejes de comunicaciones de cabotaje asociados a la producción ganadera y minera;

- 1960 a 2000: situación de encrucijada de ejes de comunicaciones a la producción minera, la administración pública y el turismo;

- 2000 a la actualidad: situación de encrucijada de ejes de comunicaciones asociados a la producción minera y la administración pública. 
Asimismo, para Cáceres, Visciglia de Gorez y Vázquez (1996) al estar situada al sureste de la provincia de Santa Cruz y del país, su posición es austral, extrema y periférica.

En relación a la Sección B de la Circunscripción IV (Fig. 2) se extiende al S-SO de la ciudad, estructurada por la Av. Asturias que coincide con el límite oriental. Como límites $\mathrm{N}$ en el centro y este la Autovía 17 de Octubre, y hacia el O el terraplén de la ex vías de Ferrocarril Río Turbio. El límite O coincide con el antiguolímite occidental del ejido municipal "la Avenida Asturias estructura este sector en el cual las chacras improductivas han sido loteadas y tienen una ocupación incipiente [...] Si bien, el sector cuenta con los servicios básicos de luz, gas, agua, pavimento y educación, carece de equipamiento recreativo, deportivo, salud y de seguridad." (Cáceres et al., 2013, p. 57)
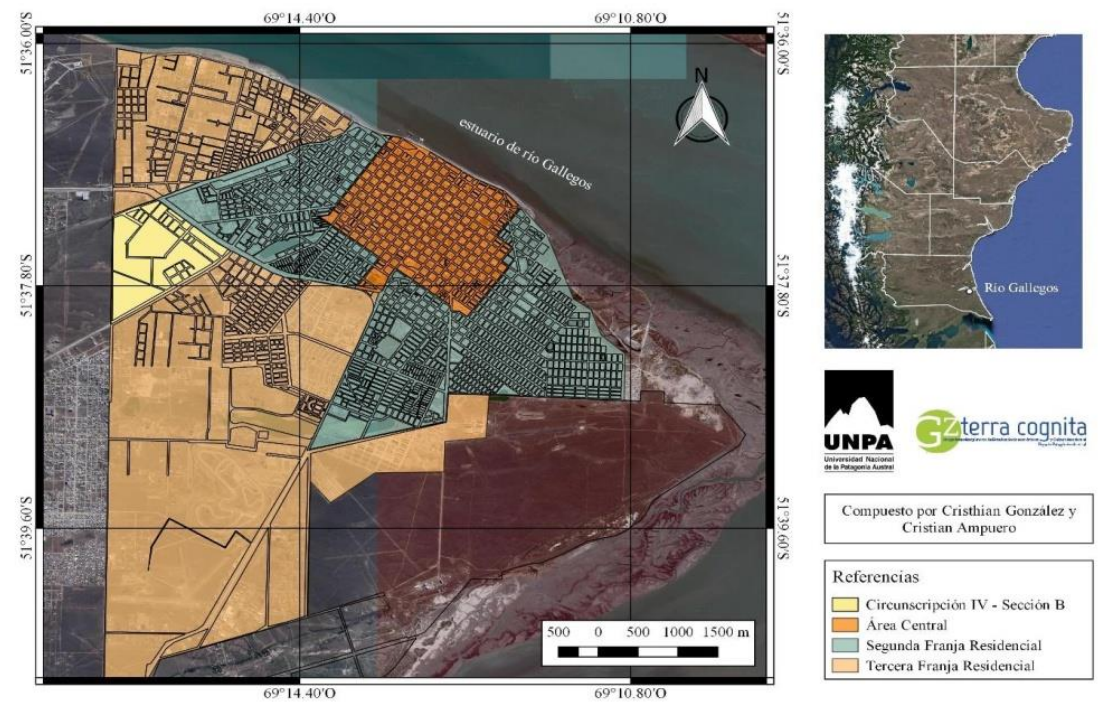

Fig. 2. Ubicación de la Circunscripción IV - Sección B en relación a la Estructura Urbana Internade Río Gallegos. Elaboración propia, modificado de Cáceres et al (2013), tomando como base imagen satelital de Google Earth

\section{MARCO TEÓRICO}

El estudio se enmarca en la Geografía Urbana, la cual es una rama de la Geografía que "considera los procesos económicos, sociales y políticos, en relación a un solo fenómeno: la ciudad”. (Carter, 1983, p. 17)

La importancia de que sea ésta la rama de la Geografía que sirva como sustento teórico para el objeto de esta investigación radica en que la misma permite comprender la dinámica y la organización ropia de la conformación de las ciudades. En términos de Cáceres (2000) para arribar el estudio desde la Geografía Urbana es pertinente acudir a las conceptualizaciones de la Estructura Urbana Interna - que alude a las actividades, infraestructura, y servicios al interior de una localidad - y el Paisaje Urbano relacionado al medio natural.

Previo al desarrollo del Paisaje Urbano de la ciudad de Río Gallegos, se torna pertinente acudir al concepto de ciudad, que según Cáceres (2000), haciendo referencia a las ciudades santacruceñas, considera que es “... un espacio geográfico muy dinámico y complejo; estos rasgos se acentúan a medida que aumenta la diversidad de sus componentes sociales, culturales, económicos, políticos y ambientales. La importante particularidad del territorio santacruceño la constituye la existencia de localidades escasamente pobladas con grandes distancias entre sí, inmersas en un marco natural imponente. Esto hace que su existencia sea 
singular y que no puedan ser comparadas con otras realidades territoriales del país. Por lo expuesto, resulta difícil encontrar una definición satisfactoria y por ende definir si las localidades santacruceñas son ciudades o no. No cabe dudas que por las características anteriores consideradas tienen una serie de servicios en lo educativo, asistencial, recreativo que les da el carácter urbano". (Cáceres, 2000, p. 870)

Respecto al Paisaje Urbano, Cáceres (2000) manifiesta que para su caracterización es necesario recurrir al estudio de la escala intra-urbana, analizando las formas urbanas o morfología, la dinámica interna de las ciudades y la ciudad en sí como un área, y deben de tenerse en cuenta tres componentes, a saber: el plano, los usos del suelo y la edificación. La autora sostiene que al conjugarse estos tres componentes tiene como resultado el paisaje urbano: "Ésta es la imagen visual, el lenguaje espacial que expresa estilos de vida, modelos y condiciones de organización socioeconómica que se han sucedido a través del tiempo".(Zárate Martín, 1991, p. 73)

Es importante destacar que el paisaje urbano puede ir transformándose a lo largo de los años, siendo el uso del suelo el que más varía, y también la edificación, pero en menor medida por todo el tiempo que implica el proceso de construcción. El tipo de plano es el que más perdura en el tiempo en virtud de la inversión que se realiza para la delimitación de las Circunscripciones, manzanas y parcelas, formando parte de la morfología urbana de un territorio.

El área de estudio se corresponde con un tipo de plano ortogonal, el cual se caracteriza por su trazado en formas regulares de manzanas con una tendencia a que las calles se crucen formando un ángulo recto. Cáceres (2000) sostiene que estos tipos de plano "han sido utilizados tanto para fundar ciudades como para el crecimiento de las mismas. Denotan una planificación previa, aunque no significa que esté bien hecha".(Cáceres, 2000, p. 876)

En cuanto al plano de la Circunscripción IV - Sección B, se observa que en dirección al Norte la forma del trazado es en forma de cuadrados y rectángulos que hacen a un tipo de plano ortogonal. En dirección al Sur, se observa que, si bien no hay una simetría en cuanto de las figuras geométricas y la relación entre ellas, respetan la forma de cuadrado y rectángulo.

Respecto a los usos del suelo, Cáceres (2000) citando los aportes de Zárate (1984) sobre los usos del suelo, los caracteriza de la siguiente manera: "Los usos del suelo expresan las diferentes utilizaciones que se hacen del espacio en función de las necesidades y actividades de la población que vive y trabaja en la ciudad" (Zárate, 1984, p. 60)

Entre los diversos usos, ligados a las funciones que se particularizan en la apropiación/transformación del suelo por parte de la población, pueden mencionarse los siguientes:

- Administrativo: agrupa organismos ligados a servicios públicos de índole asistencial, educativo y gubernamental.

- Comercial: está ligado a la concentración de la actividad comercial de diversos rubros y suele estar ubicado en el centro de la ciudad.

- Residencial: suele ser el uso de mayor superficie en las ciudades, es el espacio en el cual el hombre reside, se constituye como antagonismo al lugar de trabajo. Cuando no se trabaja, se reside: lo que se conoce como "el hogar" 
- Industrial: refiere a aquellos establecimientos en los cuales se realiza alguna actividad ligada a alguna etapa del proceso industrial, como, por ejemplo, en la provincia de Santa Cruz.

Los usos del suelo que predominan en el área de estudio son el residencial, con un mínimo de uso comercial e industrial.

En relación a la edificación, la construcción urbana según Cáceres (2000) se conoce a partir del estudio de tres elementos: el entramado, la tipología de los edificios y el volumen de la edificación.

Se destaca la tipología de los edificios en la región patagónica por ciertas características comunes de las construcciones a lo largo de la historia. Sobre la tipología patagónica, la misma autora sostiene que:

"El material de construcción predominante era la chapa; los techos a dos aguas en relación al clima húmedo del invierno - pintados con colores llamativos, no sólo para romper la monotonía del paisaje, sino también, para una mayor visibilidad en la época de nevadas. Los ambientes de estancia permanente cocina y comedor - se diseñan hacia el norte con grandes ventanales para mayor aprovechamiento de la luz y calor del sol. Las aberturas de madera preferentemente y vidrio repartido, también eran pintados con el mismo color del techo." (Cáceres, 2000, p. 883)

En lo referente al concepto de barrio, Triviño (2011) contempla los aportes de autores de diferentes disciplinas para arribar a su conceptualización desde una mirada más amplia en cuanto a este fenómeno. Entre ellos se pueden destacar:

Desde el campo de la antropología, Gravano y Guber (1991) aporta el concepto de la identidad barrial o "lo-barrial" dando cuenta que este fenómeno es mucho más complejo que lo físico propiamente dicho, puesto que en éste espacio se inscribe la dinámica social y las prácticas de los sujetos que se constituyen en ellos, siendo para el autor un lugar común en la ideología los ciudadanos, teniendo en cuenta su eficacia para hacer mención a una manera sintética que comprende diversos aspectos de la realidad.

Desde el campo de la sociología, Ander-Egg (1995) sostiene que el barrio comprende una zona o espacio en la ciudad con límites físicos y simbólicos. No obstante, cabe destacar que esos límites no necesariamente se corresponden con la división administrativa de la ciudad, puesto que son sus integrantes los que crean límites a partir de su percepción de la realidad.

Al respecto Zamorano (1985) manifiesta que los barrios pueden ser conceptualizados como secciones de la ciudad con identidad propia, la cual es atribuida en su carácter de uso común del espacio físico y simbólico, por lo cual la ciudad queda divida en sectores homogéneos que comparten un destino en común.

Por su parte, Lynch (1959) destaca que son sectores ubicados en las ciudades que son identificables por los propios habitantes y reciben un nombre. Este autor incorpora a la visión del concepto la percepción, entrando en juego la otredad, por lo cual los mismos habitantes de un barrio pueden tener una visión acerca de lo propio, como así también quienes se encuentran por fuera del barrio pueden tener otra visión del mismo: se construyen así sectores en la ciudad con identidad propia. 
Concluyendo con la conceptualización de barrio, se torna pertinente acudir a los aportes de Cáceres (2000) quien desde la óptica de la Geografía Urbana considera que el barrio se encuentra comprendido desde dos dimensiones: la identidad propia de sus habitantes, y la forma y función del mismo. La forma se relaciona con lo observable del sector, y la función está ligada al tipo de uso y apropiación que se haga del suelo.

Al hacer una revisión sobre la literatura existente sobre el tema, se pone en evidencia que al trabajar con esta categoría debe de contemplarse para captar la esencia de los procesos intrínsecos a la conformación de los mismos, no sólo lo referente a lo físico-arquitectónico, sino también aquellas prácticas cotidianas de los vecinos que dan cuenta de su identidad como barrio, de la identidad barrial construida en su historia como vecinos.

A lo largo de este trabajo, la forma y la función del barrio son dos ejes centrales para el reconocimiento y espacialización de Unidades Territoriales Residenciales (UTR).

En cuanto a su forma, se analizan variables tales como el proceso de ocupación del suelo (consolidado o incipiente), viviendas unifamiliares o multifamiliares, y el entramado (abierto o cerrado).

En cuanto a la función, se analizan los usos del suelo. Si bien su nombre lo indica, el uso es residencial, pero puede ocurrir que en algunas de ellas existan otros usos del suelo, tales como el comercial, recreativo o de servicios.

Por último, se define que una Unidad Territorial Residencial es un espacio que demuestra homogeneidad y cohesión territorial particularmente por constituirse como un área en donde predomina el uso del suelo residencial y que presenta características geográficas, históricas, arquitectónicas y sociales que le otorgan uniformidad.

\section{MATERIALES Y MÉTODOS}

La metodología que le dio encuadre y sustentó al presente trabajo es cuantitativa, puesto que el reconocimiento y espacialización de Unidades Territoriales Residenciales (UTR) implicó su cuantificación, y asimismo, la correlación de diversas variables (usos del suelo, proceso de ocupación del suelo, entramado y tipología de vivienda) que éstas comprenden.

En cuanto a las ramas del enfoque cuantitativo, el trabajo fue descriptivo en virtud de que, en un primer momento, en los resultados se dio cuenta de las características de las UTR, y explicativo puesto que a partir de la correlación de las variables en la síntesis y en la discusión se pudo explicar la conformación de las UTR en el área de estudio.

Se realizó en un primer momento un relevamiento y lectura de bibliografía específica para la construcción del marco teórico conceptual, para lo cual se tomaron aportes de la Geografía Urbana, la cual comprende el estudio del Paisaje Urbano y la Estructura Urbana Interna. En el marco de ello se conceptualizó Unidad Territorial Residencial, aporte significativo que genera este trabajo.

Posteriormente, se realizó el relevamiento de información técnica sobre la Circunscripción IV - Sección B, compuesto por documentos tales como ordenanzas municipales y cartografía antigua provista por el Archivo Histórico Municipal, fotografías aéreas históricas e imágenes satelitales actuales a los fines de profundizar el conocimiento del área de estudio, y se delimitaron Unidades Territoriales Residenciales (UTR) de manera provisoria. 
Se realizaron dos salidas de campo. En la primera de ellas se logró el primer acercamiento al área de estudio, para ejercitar el reconocimiento, desde el campo disciplinar de la Geografía, de la Estructura Urbana Interna y el Paisaje Urbano de Río Gallegos: el área urbana y rururbana intra y extra-ejidal; el centro, la segunda y tercera franja residencial; la división en circunscripciones, manzanas, y lotes; el tipo de plano, usos del suelo y edificación. El objetivo de esta salida radicó en un conocimiento general que permitió el reconocimiento del área de estudio enmarcado en un fenómeno aún más grande: la ciudad. También permitió junto con la lectura de bibliografía específica, el diseño de una planilla de relevamiento para la segunda salida de campo.

La segunda salida de campo, consistió en un recorrido por la totalidad de la Circunscripción IV - Sección B, y en tanto insumo técnico-instrumental se utilizó la planilla de relevamiento diseñada previamente en gabinete para la carga de información proveniente de la observación directa, datos de coordenadas geográficas con GPS, y también se tomaron fotografías.

Finalmente, se realizó el trabajo de gabinete, donde se digitalizaron las UTR en un Sistema de Información Geográfica (SIG), a las cuales se les asignó una letra del alfabeto siguiendo el sentido de las agujas del reloj. El software utilizado para la elaboración de cartografía temática fue el QGIS en su versión 3.2. "Bonn".

Se destaca que para la elaboración de la cartografía se recibió formación específica en cartografía

\section{RESULTADOS}

Los puntos extremos del Sitio de la Sección B de la Circunscripción IV son:

- Norte: $51^{\circ} 37^{\prime} 9.46^{\prime \prime}$ LS y $69^{\circ} 15^{\prime} 77^{\prime}$ ' LO

- Este: $51^{\circ} 37^{\prime} 31.57^{\prime}$ 'LS y $69^{\circ} 15^{\prime} 10.02$ ' ' LO

- Sur: Latitud $51^{\circ} 36^{\prime} 3.85^{\prime \prime}$ LS y $69^{\circ} 16^{\prime} 26.08^{\prime}$ ' LO

- Oeste: $51^{\circ} 37^{\prime} 16.36^{\prime \prime}$ LS y $69^{\circ} 16^{\prime} 25^{\prime \prime}$ LO

En cuanto al Emplazamiento, el área de estudio es atravesado desde Este a Oeste por un paleocauce de $11 \mathrm{~m}$. de profundidad (Fig. 3) siendo la continuidad de la Laguna María la Gorda. 


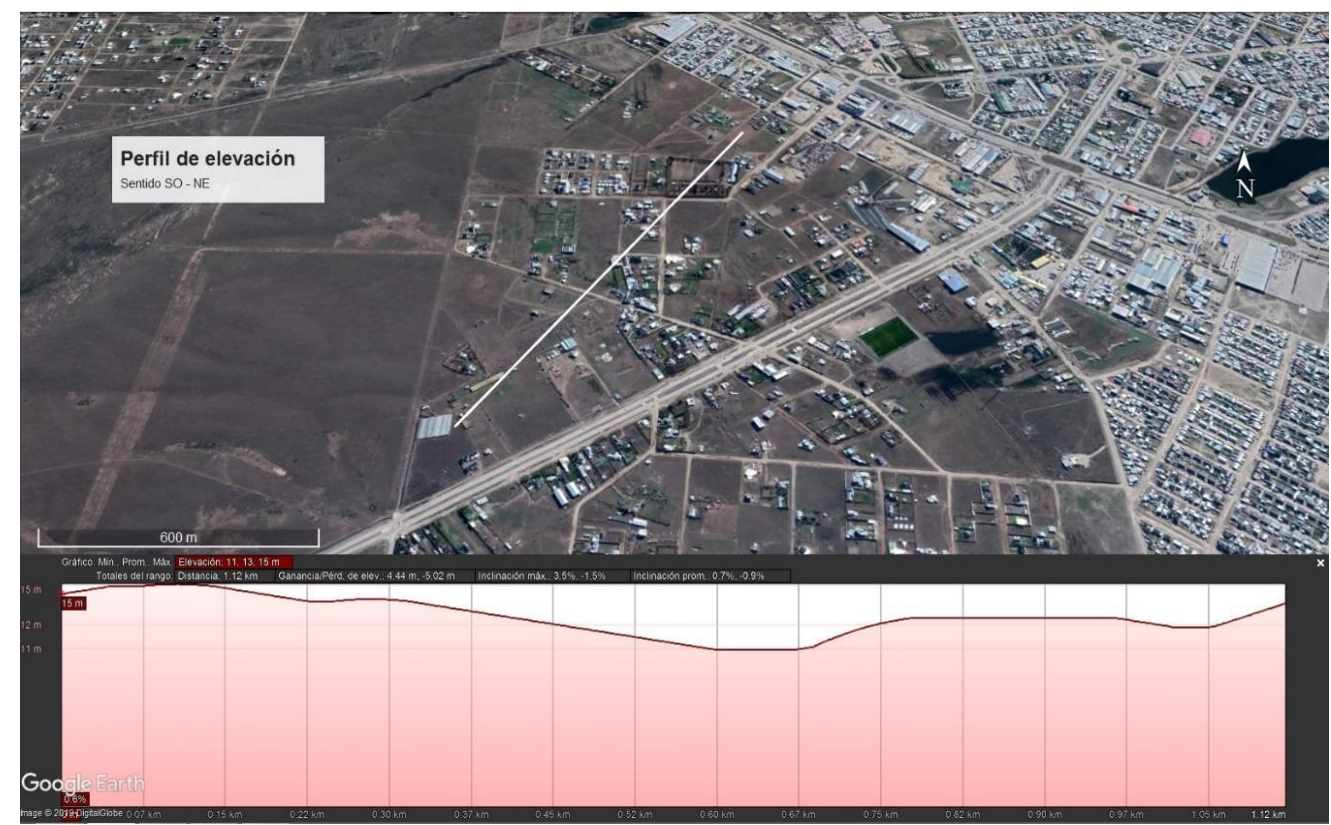

Fig. 3. Perfil de elevación de la Circunscripción IV - Sección B. Elaborado con Google Earth

La Situación es extrema con respecto a la ciudad, se comunica con el resto de Río Gallegos a través de la Avenida Asturias y la Autovía 17 de Octubre.

En cuanto a la Estructura Urbana Interna, el área de estudio comprende una superficie aproximada de $1.39 \mathrm{~km}^{2}$. Pertenece a la TFR el sector Norte - comprendido entre las calles Autovía 17 de Octubre, Yugoslavia, Avenida Asturias y ex terraplén del ramal ferroviario Río Turbio - Límite con la Sección A de la Circunscripción IV: corresponde a la UTR A encontrándose consolidada, con una predominancia del uso residencial, con otros usos como comercial y recreativo. El de depósitos y estación de servicios, quedan excluidos de esta UTR.

El resto de la Sección, corresponde al Área RururbanaIntraejidal (ARI), donde chacras improductivas han sido loteadas y venidas para el uso residencial, con servicios básicos de energía, gas en red, pero con ausencia de un Nodo de Servicios.

A partir del trabajo de campo, se reconocieron y espacializaron un total de 9 (nueve) Unidades Territoriales Residenciales en la Circunscripción IV - Sección B (Fig. 4 y Tabla 1) teniendo en cuenta los elementos que constituyen el Paisaje Urbano del área de estudio, presentados en las tablas 2, 3, 4 y 5 . 


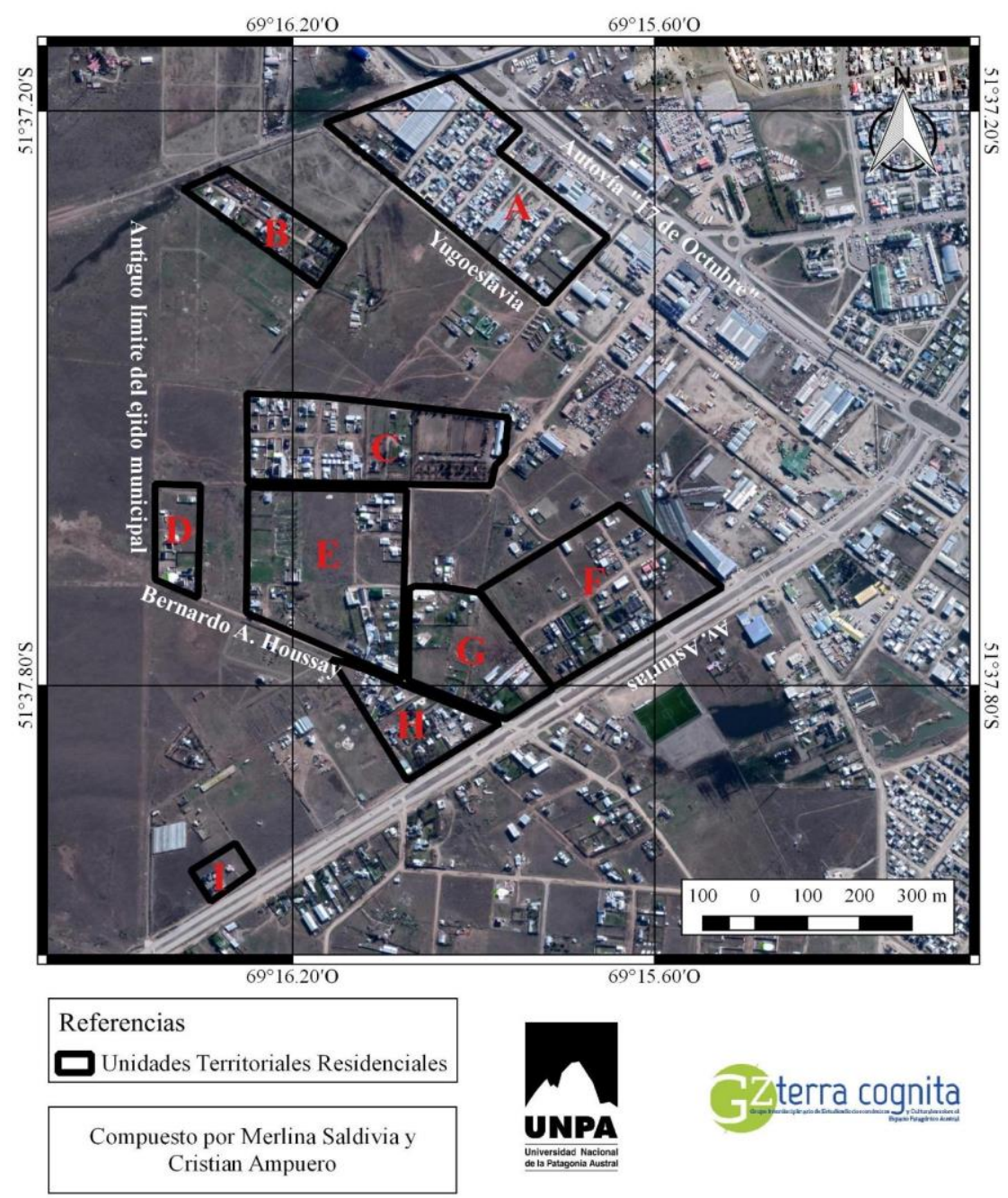

Fig. 4. Circunscripción IV, Sección B - Unidades Territoriales Residenciales (UTR). Elaboración propia, tomando como base la imagen satelital de Google Earth

Tabla 1. Límites de las UTR. Elaboración propia con datos relevados por los autores

\begin{tabular}{|c|c|c|c|c|}
\hline \multirow{2}{*}{$\begin{array}{c}\text { Unidades } \\
\text { Territoriales } \\
\text { Residenciales }\end{array}$} & \multicolumn{4}{|c|}{ Límites } \\
\hline & Norte & Este & Sur & Oeste \\
\hline $\mathrm{A}$ & $\begin{array}{l}\text { Paseo de los } \\
\text { Arrieros y el Notro }\end{array}$ & E. Fernández & Yugoslavia & $\begin{array}{c}\text { Límite con la } \\
\text { Sección A de la } \\
\text { Circunscripción IV }\end{array}$ \\
\hline B & Calle $\mathrm{S} / \mathrm{N}$ & Los Sauces & $\begin{array}{c}\text { Límite con la } \\
\text { Sección A de la } \\
\text { Circunscripción IV }\end{array}$ & Calle $\mathrm{S} / \mathrm{N}$ \\
\hline $\mathrm{C}$ & Calle S/N & Don Eusebio Ilhero & $\begin{array}{l}\text { Néstor Osvaldo } \\
\text { Forlón }\end{array}$ & Alberto J. Bark \\
\hline $\mathrm{D}$ & $\begin{array}{l}\text { Néstor Osvaldo } \\
\text { Forlón }\end{array}$ & Calle $\mathrm{S} / \mathrm{N}$ & $\begin{array}{c}\text { Bernardo A. } \\
\text { Houssay }\end{array}$ & $\begin{array}{l}\text { Antiguo límite del } \\
\text { Ejido Municipal }\end{array}$ \\
\hline $\mathrm{E}$ & $\begin{array}{l}\text { Néstor Osvaldo } \\
\text { Forlón }\end{array}$ & Río Chico & $\begin{array}{l}\text { Bernardo A. } \\
\text { Houssay }\end{array}$ & Calle $\mathrm{S} / \mathrm{N}$ \\
\hline $\mathrm{F}$ & Calle S/N & Avenida Asturias & Calle S/N & Calle S/N \\
\hline G & Calle $\mathrm{S} / \mathrm{N}$ & Avenida Asturias & $\begin{array}{l}\text { Bernardo A. } \\
\text { Houssay }\end{array}$ & Río Chico \\
\hline $\mathrm{H}$ & $\begin{array}{c}\text { Bernardo A. } \\
\text { Houssay }\end{array}$ & Avenida Asturias & - & - \\
\hline I & - & Avenida Asturias & - & - \\
\hline
\end{tabular}


Tabla 2. Usos del Suelo. Elaboración propia con datos relevados por los autores

\begin{tabular}{cccc}
\hline Unidades Territoriales & & Usos del Suelo & \\
\cline { 2 - 4 } Residenciales & Residencial & Comercial & \\
A & $\mathrm{X}$ & $\mathrm{X}$ & \\
$\mathrm{B}$ & $\mathrm{X}$ & & \\
$\mathrm{C}$ & $\mathrm{X}$ & & \\
$\mathrm{D}$ & $\mathrm{X}$ & $\mathrm{X}$ & \\
$\mathrm{E}$ & $\mathrm{X}$ & & \\
$\mathrm{F}$ & $\mathrm{X}$ & & \\
$\mathrm{G}$ & $\mathrm{X}$ & & \\
$\mathrm{H}$ & $\mathrm{X}$ & & \\
$\mathrm{I}$ & $\mathrm{X}$ & & \\
\hline
\end{tabular}

Tabla 3. Proceso de ocupación. Elaboración propia con datos relevados por los autores

\begin{tabular}{ccc}
\hline Unidades Territoriales & \multicolumn{2}{c}{ Proceso de Ocupación del Suelo } \\
\cline { 2 - 3 } Residenciales & Consolidado & Incipiente \\
\cline { 2 - 3 } A & $\mathrm{X}$ & $\mathrm{X}$ \\
$\mathrm{B}$ & $\mathrm{X}$ & $\mathrm{X}$ \\
$\mathrm{C}$ & & $\mathrm{X}$ \\
$\mathrm{D}$ & & $\mathrm{X}$ \\
$\mathrm{E}$ & $\mathrm{X}$ & $\mathrm{X}$ \\
$\mathrm{F}$ & & $\mathrm{X}$ \\
G & & \\
H & & \\
$\mathrm{I}$ & & \\
\hline
\end{tabular}

Tabla 4. Entramado. Elaboración propia con datos relevados por los autores

\begin{tabular}{|c|c|c|}
\hline \multirow{2}{*}{$\begin{array}{c}\text { Unidades Territoriales } \\
\text { Residenciales }\end{array}$} & \multicolumn{2}{|c|}{ Entramado } \\
\hline & Abierto & Cerrado \\
\hline $\mathrm{A}$ & & $\mathrm{X}$ \\
\hline B & $X$ & \\
\hline $\mathrm{C}$ & $X$ & \\
\hline D & $X$ & \\
\hline $\mathrm{E}$ & $X$ & \\
\hline $\mathrm{F}$ & $\mathrm{X}$ & \\
\hline G & $X$ & \\
\hline $\mathrm{H}$ & $X$ & \\
\hline I & $X$ & \\
\hline
\end{tabular}

Tabla 5. Tipo de vivienda / Tipología arquitectónica. Elaboración propia con datos relevados por los autores

\begin{tabular}{cccc}
\hline Unidades Territoriales & \multicolumn{2}{c}{ Tipo de Vivienda } & Tipología Patagónica \\
\cline { 2 - 4 } Residenciales & Unifamiliar & Multifamiliar & SI - NO \\
\hline A & X & & SÍ \\
B & $\mathrm{X}$ & & SÍ \\
C & $\mathrm{X}$ & $\mathrm{X}$ & SÍ \\
$\mathrm{D}$ & & $\mathrm{X}$ & SÍ \\
$\mathrm{E}$ & & & NO \\
$\mathrm{F}$ & $\mathrm{X}$ & & SÍ́ \\
$\mathrm{G}$ & $\mathrm{X}$ & $\mathrm{X}$ & SÍ \\
$\mathrm{H}$ & & & SÍ \\
$\mathrm{I}$ & $\mathrm{X}$ & SÍ
\end{tabular}


A partir de la correlación existente entre las variables presentadas en las tablas de análisis, es decir, el uso del suelo, el proceso de ocupación, el entramado, y el tipo de vivienda / tipología arquitectónica, se analizan las Unidades Territoriales Residenciales (UTR) identificadas:

Unidad Territorial Residencial A: el proceso de ocupación del suelo es consolidado, predomina el uso del suelo residencial con viviendas unifamiliares de entramado cerrado y tipología patagónica, aunque en algunos lotes se observa la presencia de diversos tipos de viviendas. Cabe aclarar que la presencia de otros usos del suelo es mínima, entre los que se menciona el uso comercial.

Unidad Territorial Residencial B: el proceso de ocupación está en proceso de consolidación, predomina el uso residencial con una mayoría de viviendas unifamiliares con entramado abierto, y tipología patagónica. Cabe destacar que en esta UTR se encuentra una vivienda de entramado cerrado.

Unidad Territorial Residencial C: el proceso de ocupación es incipiente, predomina el uso residencial con mayoría de viviendas unifamiliares con entramado abierto. En esta UTR se encuentra la Chacra Estevez.

Unidad Territorial Residencial D: el proceso de ocupación es incipiente, predomina el uso residencial con viviendas multifamiliares de entramado abierto, y tipología patagónica. Cabe destacar que hay una escasa presencia de viviendas unifamiliares.

Unidad Territorial Residencial E: el proceso de ocupación es incipiente, predomina el uso residencial con viviendas multifamiliares de entramado abierto. Cabe destacar que hay una escasa presencia de viviendas unifamiliares y otros usos del suelo: comercial e industrial.

Unidad Territorial Residencial F: el proceso de ocupación es consolidado, predomina el uso residencial con viviendas unifamiliares de entramado abierto, y tipología patagónica.

Unidad Territorial Residencial G: el proceso de ocupación es incipiente, predomina el uso residencial, con viviendas unifamiliares de entramado abierto, y tipología patagónica.

Unidad Territorial Residencial H: el proceso de ocupación es incipiente, predomina el uso residencial con viviendas multifamiliares de entramado abierto, y tipología patagónica.

Unidad Territorial Residencial I: el proceso de ocupación es incipiente, predomina el uso residencial con viviendas unifamiliares con entramado abierto, y tipología patagónica.

De la correlación de las variables identificadas en las UTR, se presenta el siguiente mapa síntesis (Fig. 5). 


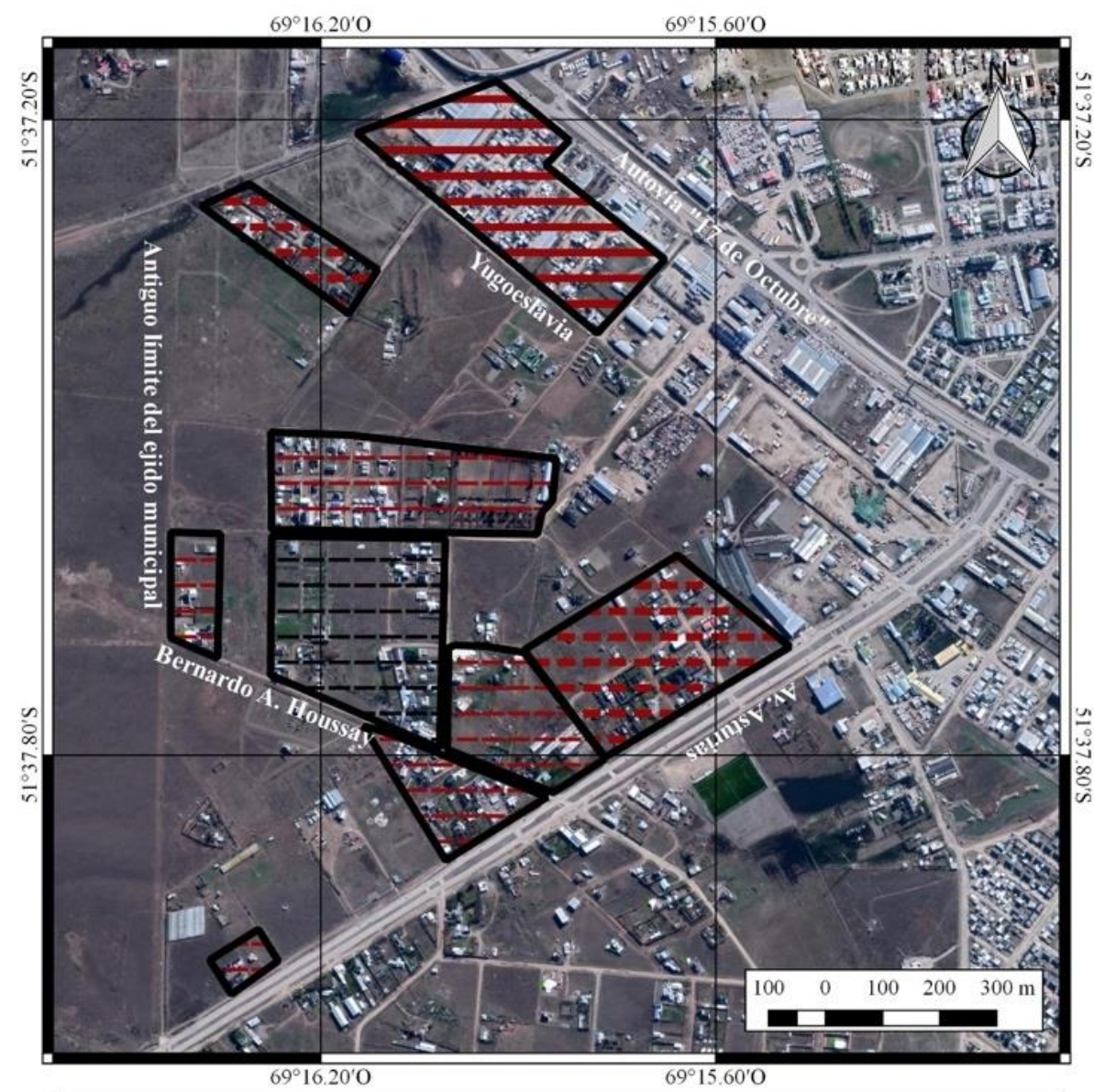

\begin{tabular}{|l|}
\hline Leyenda \\
Proceso de ocupación, entramado y tipologia arquitectónica en las UTR \\
- Proceso de ocupación consolidado, entramado cerrado con tipologia patagónica \\
- - Proceso de ocupación consolidado, entramado abierto con tipología patagónica \\
-- Proceso de ocupación incipiente, entramado abierto con tipologia patagónica \\
\hline Proceso de ocupación incipiente, entramado abierto con tipologia no patagónica
\end{tabular}

Figura 5. Proceso de ocupación, entramado y tipología arquitectónica en las UTR. Elaboración propia, tomando como base la imagen satelital de Google Earth

A modo de síntesis, se plantea que la confluencia de la Avenida Asturias y Autovía 17 de Octubre actúan como eje de fijación para el proceso de ocupación del suelo en términos de uso residencial: en tanto en la UTR A el proceso es continuo, en las UTR pertenecientes al Área RurbanaIntraejidal (ARI) es discontinuo. Por tal motivo, se afirma que en la Circunscripción IV - Sección B, el proceso de ocupación es consolidado al NE y, en la medida que se transita hacia el SO, el proceso de ocupación es incipiente.

Del análisis del área de estudio se desprende que hay una tendencia al tipo de vivienda unifamiliar y de tipología patagónica. Asimismo, se destaca la predominancia en gran parte de la Sección del entramado abierto, lo cual se relaciona al proceso de ocupación incipiente y discontinuo. No obstante, la excepción a tal afirmación es la UTR A, puesto que la totalidad de los lotes se encuentran ocupados, con la presencia de entramado cerrado. 


\section{CONCLUSIONES}

En virtud de lo expuesto, el objetivo de esta investigación de reconocer y espacializar Unidades Territoriales Residenciales (UTR), efectivamente se logró al haberse delimitado y caracterizado un total de nueve UTR, abordadas desde el estudio de la Geografía Urbana, teniendo en cuenta dos ejes: la Estructura Urbana Interna (TRF - ARI) y el Paisaje Urbano (tipo de plano, usos del suelo y edificación).

Respecto al aporte de esta investigación, se destaca que el reconocimiento y espacialización de UTR, en tanto categoría analítica, permite comprender desde su complejidad el uso y apropiación del suelo por parte de sujetos históricos, atribuyendo significaciones a un territorio a partir de sus prácticas cotidianas.

Se destaca que los resultados obtenidos aportan parcialmente al PI 29A/380PDTS, sobre marco teórico y metodología, al permitir el conocimiento de las áreas ocupadas con usos residenciales, para la posterior zonificación y terrenos disponibles para la proyección urbanística del municipio.

\section{AGRADECIMIENTOS}

Al Consejo Interuniversitario Nacional y a la Universidad Nacional de la Patagonia Austral por financiar las Becas Estímulo a las Vocaciones Científicas para estudiantes de grado, en el marco de la cual se realizó este trabajo.

Al Proyecto de Investigación 29A/PDTS380 “Tercera Franja Residencial de Río Gallegos: Diagnóstico territorial de áreas con ausencia y presencia de cobertura de servicios. Propuesta de localización de Nodos de Servicios", por el financiamiento de las salidas de campo.

\section{BIBLIOGRAFÍA}

ANDER-EGG, E. (1992): Técnicas en Investigación Social. Editorial Hvmanitas. Buenos Aires.

AMPUERO, C.; CÁCERES, A. P.; NORAMBUENA, M., FRIAS, P.; SEGOVIA, M.; MELGAREJO, G. (2018): Diagnóstico Territorial de la Tercera Franja Residencial de Río Gallegos, Patagonia Austral Argentina. Aportes de Becarios Estudiantes de la UNPA. Río Gallegos. Revista Contribuciones Científicas. Vol. 30. GÆA, Sociedad Argentina de Estudios Geográficos. Buenos Aires.

CÁCERES A. P.; GARCÍA, A. N. (2004): Diferenciación interna del espacio Geográfico de Río Gallegos: Estudio Preliminar. IGEOPAT. Párrafos geográficos. Año II Nº 3.

CÁCERES, A. P; SÁENZ, J. L; MELGAREJO, G.; AMPUERO, C. (2011): Definición de estratos residenciales en el Área RururbanaIntraejidal de Río Gallegos; Ciudad intermedia de la Patagonia Austral Argentina. II Congreso Nacional de Departamentos de Geografía de Universidades Públicas. Santa Fe.

CÁCERES, A. P. (2000): Estructura y paisajes urbanos de Santa Cruz. El Gran Libro de Santa Cruz. Alfa Centro Literario y Editorial Oriente. 
CÁCERES, A. P. (2013): Crecimiento urbano de la ciudad de Río Gallegos. Capital de la provincia de Santa Cruz, Patagonia Austral Argentina (1985 - 2010). Revista $\begin{array}{llll}\text { Científica } & \text { de } & \text { Geografía } & \mathrm{N}^{\circ}\end{array}$ https://www.usal.edu.ar/archivos/geousal/docs/crecimiento_urbano_rio_gallegos_188 $\underline{\text { 5-2010.pdf }}$

CÁCERES, A. P., VISCIGLIA DE GOREZ, E., y VÁZQUEZ, M. (1996): La Percepción Urbana de Río Gallegos. Anales GÆA Sociedad Argentina de Estudios Geográficos. Tomo XX. Buenos Aires.

CÁCERES, A. P.; NORAMBUENA, M. V.; AMPUERO, C.; TRIVIÑO, G. (2013): Consolidación de la tercera franja residencial de Río Gallegos, Patagonia Argentina. Contribuciones Científicas Vol. 25, Sociedad Argentina de Estudios Geográficos GÆA. Buenos Aires.

CÁCERES, A. P.; SEGOVIA, M.; SOTO, J; NORABUENA, M; FRIAS, P.; AMPUERO, C. (2016): Posición geográfica de Río Gallegos en la Patagonia Austral. Actas científicas del Congreso Internacional de Geografía - $77^{\circ}$ Semana de la Geografía. GÆA, Sociedad Argentina de Estudios Geográficos. Buenos Aires.

CÁCERES, A.; AMPUERO, C. (2008): Análisis de la distribución espacial de nodos de servicios en la segunda franja residencial de una ciudad intermedia patagónica como aporte al diagnóstico territorial. Boletín geográfico. Año XXX, N³1. Edición especial: VII Jornadas Patagónicas de Geografía.Departamento Geografía, Universidad Nacional del Comahue. Neuquén.

CARTER, H. (1983): El estudio de la geografía urbana. Instituto de Estudios de Administración Local. Madrid.

EXPEDIENTE $\mathrm{N}^{\circ}$ 13.520-1960. Acumulado $\mathrm{N}^{\circ}$ 15.233-1961. Río Gallegos. 9 de Julio de 1962.

GONZÁLEZ, C.; CÁCERES, A. P. (2018): Determinación del área de influencia del Nodo de Servicio (NS) de la Circunscripción III, Tercera Franja Residencial (TFR) de Río Gallegos. Informe final de beca de investigación. ICT-UNPA. Río Gallegos.

GRAVANO, A. y GÚBER, R (1991): Barrio sí, villa también. Biblioteca Política Argentina. Buenos Aires.

LYNCH, K. (1959): La imagen de la ciudad. Editorial Infinito. Buenos Aires.

MUNICIPALIDAD DE RÍO GALLEGOS (2013): Ordenanza $\mathrm{N}^{\circ} 3259 / 98$ y modificatorias 3864/00; 3964/00; 4003/00; 4538/02; 4566/02; 4731/02; 4997/03; 5160/03; 5182/03; 5188/03; 6191/07; 6406/07; 6536/08; 6576/08; 6597/08; 6705/09. Río Gallegos.

PORTAL OFICIAL DEL INSTITUTO NACIONAL DE ESTADÍSTICA Y CENSO DE LA REPÚBLICA ARGENTINA (2018): Censo de 2010. Disponible en: https://www.indec.gob.ar/

REAL ACADEMIA ESPAÑOLA, RAE (2018): Diccionario enciclopédico de la lengua española. Disponible en: http://www.rae.es/

TRIVIÑO, G.; CÁCERES, A. P. (2011): Análisis y caracterización sociodemográfica de asentamientos en la Segunda Franja Residencial de la ciudad de Río Gallegos. El caso particular del asentamiento "Madres a la Lucha". ICT-UNPA-32-2011.

ZAMORANO, M. (1992): Geografía Urbana. Colección Geográfica. Editorial Ceyne. Buenos Aires.

ZÁRATE, A. (1984): El mosaico urbano. Organización interna y vida en las ciudades. Editorial Cincel. Madrid.

ZÁRATE MARTÍN, A. (1991): El espacio interior de las ciudades. Editorial Síntesis. Madrid. 